\title{
Inhibition of the NLRP3 inflammasome provides neuroprotection in rats following amygdala kindling-induced status epilepticus
}

Xiang-Fei Meng ${ }^{1}$, Lan Tan ${ }^{1,2,3^{*}}$, Meng-Shan Tan ${ }^{3}$, Teng Jiang ${ }^{4}$, Chen-Chen Tan ${ }^{1}$, Meng-Meng Li ${ }^{1}$, Hui-Fu Wang ${ }^{2}$ and Jin-Tai $Y u^{1,2,5^{*}}$

\begin{abstract}
Background: NLRP3 inflammasome is proposed to regulate inflammation in several neurological diseases, but its role in epilepsy remains largely unknown. This study aimed to investigate the role of the NLRP3 inflammasome in neuroinflammation, spontaneous recurrent seizures (SRS) and hippocampal neuronal loss in rat brain following amygdala kindling-induced status epilepticus (SE).
\end{abstract}

Methods: We detected the protein levels of IL-1 $\beta$ and NLRP3 inflammasome components by Western blot in the hippocampus of shams and SE rats at different time points following SE. To further examine whether the activation of the NLRP3 inflammasome contributes to SE-associated neuronal damage, we employed a nonviral strategy to knock down NLRP3 and caspase-1 expression in brain before undergoing SE. Proinflammatory cytokine levels and hippocampal neuronal loss were evaluated at 12 hours and at 6 weeks following SE respectively in these NLRP3 and caspase-1 deficient rats. Meanwhile, SRS occurrence was evaluated through a 4-week video recording started 2 weeks after SE in these NLRP3 and caspase- 1 deficient rats.

Results: IL-1 $\beta$ levels and NLRP3 inflammasome components levels dramatically increased at 3 hours after SE, and reached a maximum at 12 hours after SE compared with the control group. Knock down of NLRP3 or caspase-1 decreased the levels of IL-1 $\beta$ and IL-18 at 12 hours after SE, which was accompanied by a significant suppression in the development and severity of SRS during the chronic epileptic phase. Meanwhile, knock down of NLRP3 or caspase-1 led to a remarkable reduction of hippocampal neuronal loss in the CA1 and CA3 area of the hippocampus at 6 weeks after SE.

Conclusions: Our study provides the first evidence that the NLRP3 inflammasome was significantly up-regulated following SE. More importantly, we show that inhibition of the NLRP3 inflammasome provides neuroprotection in rats following SE. These findings suggest that NLRP3 may represent a potential target for the treatment of epileptogenesis

Keywords: NLRP3, Inflammasome, Status epilepticus, Cytokine, IL-1ß, IL-18, Caspase-1, Neuroinflammation, Spontaneous recurrent seizures, Hippocampal neuronal loss

\footnotetext{
* Correspondence: dr.tanlan@163.com; yu-jintai@163.com

'Department of Neurology, Qingdao Municipal Hospital, School of Medicine,

Qingdao University, Qingdao, PR China

${ }^{2}$ Department of Neurology, Qingdao Municipal Hospital, Nanjing Medical

University, Nanjing, PR China

Full list of author information is available at the end of the article
}

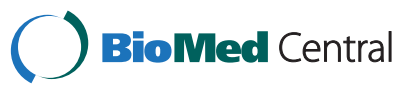

2014 Meng et al.; licensee BioMed Central. This is an Open Access article distributed under the terms of the Creative Commons Attribution License (http://creativecommons.org/licenses/by/4.0), which permits unrestricted use, distribution, and reproduction in any medium, provided the original work is properly credited. The Creative Commons Public Domain Dedication waiver (http://creativecommons.org/publicdomain/zero/1.0/) applies to the data made available in this article, unless otherwise stated. 


\section{Background}

Status epilepticus (SE), which is one of the most serious manifestations of epilepsy, can be defined as 'a condition characterized by an epileptic seizure that is so frequent or so prolonged as to create a fixed and lasting condition' [1]. Brain inflammation promotes increased neuronal excitability, decreases seizure threshold and is likely to be involved in the molecular, structural and synaptic changes characterizing epileptogenesis [2]. Among all the enhanced proinflammatory cytokines following SE, IL- $1 \beta$ is regarded as a pivotal therapeutic target in SE, as observations have demonstrated IL-1 $\beta$ as being an important inflammationrelated epileptogenic factor [3 5]. Many anti-seizure drugs are known to decrease IL- $1 \beta$ levels [6,7]. Moreover, the inhibition or deletion of caspase-1, the enzyme which cleaves pro-IL- $1 \beta$ producing the mature and biologically active form of IL- $1 \beta$, attained significant seizure reduction $[8,9]$. However, the mechanisms by which the production of IL- $1 \beta$ is regulated have not been established.

The nucleotide binding and oligomerization domainlike receptor family pyrin domain-containing 3 (NLRP3) inflammasome is a multiprotein complex that mediates the activation of caspase- 1 , which in turn cleaves pro-IL-1 $\beta$ to form the mature IL- $1 \beta$ and is found to be a pivotal mediator of IL-1 $\beta$ function [10]. The NLRP3 inflammasome [11], composed of NLR family, pyrin domain containing 3 (NLRP3), apoptosis-associated specklike protein containing a caspase recruitment domain (ASC) and caspase- 1 , mediates IL- $1 \beta$ transcription and functions via coupling with the NF- $\mathrm{KB}$ inflammatory pathway [12]. The NLRP3 inflammasome has been demonstrated as being associated with the innate immunity and inflammatory regulation of the central nervous system (CNS) [13 15]. It should be noted that the high concentrations of extracellular ATP and $\mathrm{K}^{+}$ions, the generation of reactive oxygen species (ROS), the increased intracellular $\mathrm{Ca}^{2+}$ concentration, acidosis, hypoxia and cell swelling can activate NLRP3 [16 18]. Actually, all of the above factors underlie the generation of SE [19 22]. We hypothesized that this inflammasome may have potential to induce IL-1 $\beta$-related neuroinflammation in SE. Therefore, it is intriguing to investigate the role of the NLRP3 inflammasome in SE-associated pathology and functional outcomes.

To test this hypothesis, we first investigated the expression profiles of IL-1 $\beta$ and NLRP3 inflammasome components, including NLRP3 and caspase-1 after SE. Next, we applied small interfering RNAs (siRNAs) to knock down NLRP3 and caspase-1 in vivo, and measured the alteration in proinflammatory cytokine and NLRP3 inflammasome components as well as the effects on functional outcomes.

\section{Methods}

\section{Animals and experiments groups}

To avoid the interference of estrogen on microglial activation, neuroinflammation and cognitive function [23], only male rats were used in this study. Adult male SpragueDawley (SD) rats weighing 260 to $300 \mathrm{~g}$ were obtained from the Experimental Animal Center of Qingdao University. The rats were specific-pathogen free rats, regularly checked to ensure the status, and housed in a pathogen-free room with a 12-hour light/dark cycle and given free access to food and water. All experiments were performed in strict accordance with the National Institute of Health Guide for the Care and Use of Laboratory Animals. Animal care and sacrifice were conducted according to methods approved by the Qingdao University Animal Experimentation Committee, and all efforts were made to minimize the number of animals used and their suffering. Experimental group allocation and experimental design are shown in Additional file 1: Figure S1.

\section{siRNA administration in rat brain}

The NLRP3 siRNA was designed and synthesized by Invitrogen (Carlsbad, CA, USA). Caspase-1 siRNA and control siRNA were purchased from Santa Cruz Biotechnology (Santa Cruz, CA, USA). Entranster in vivo transfection reagent was purchased from Engreen Biosystem Co, Ltd (Beijing, China). We prepared the Entranster -in vivo-siRNA mixture according to the manufacturers instructions. Briefly, $50 \mu \mathrm{g}$ siRNA were resuspended in $50 \mu \mathrm{L}$ RNase-free water to make a siRNA solution. Then, $50 \mu \mathrm{L}$ of siRNA solution were mixed with $50 \mu \mathrm{L}$ of Entranster -in vivo transfection reagent and $100 \mu \mathrm{L}$ artificial cerebrospinal fluid (aCSF, composition in $\mathrm{mmol} / \mathrm{L}: \mathrm{NaCl} 130, \mathrm{KCl} 2.99, \mathrm{CaCl}_{2} 0.98, \mathrm{MgCl}_{2} 6 \mathrm{H}_{2} \mathrm{O}$ 0.80, $\mathrm{NaHCO}_{3} 25, \mathrm{Na}_{2} \mathrm{HPO}_{4} 12 \mathrm{H}_{2} \mathrm{O} \quad 0.039, \mathrm{NaH}_{2}$ $\mathrm{PO}_{4} 2 \mathrm{H}_{2} \mathrm{O}$ 0.46) to obtain a $200 \mu \mathrm{L}$ Entranster -in vivo transfection mixture. Then, the $200 \mu \mathrm{L}$ Entranster -in vivo transfection mixture (containing NLRP3 or caspase-1 siRNA, control siRNA, no siRNA (aCSF only)) was filled into an osmotic pump (Model 2006; ALZET, Cupertino, CA, USA). Meanwhile, rats were anesthetized with $10 \%$ chloral hydrate $(0.3 \mathrm{~mL} / 100 \mathrm{~g}$, intraperitoneal) and were placed in a stereotaxic apparatus (Stoelting, Wood Dale, IL, USA). A brain-infusion cannula (Brain Infusion Kit 2; ALZET, Cupertino, CA, USA) coupled via vinyl tubing to the osmotic pump was implanted into the dorsal third ventricle (AP: $-1.8 \mathrm{~mm}$; L: $-0 \mathrm{~mm}$; V: $-5 \mathrm{~mm}$ ). The osmotic pump was placed subcutaneously between the rat scapulae, and the siRNAs were continuously infused into the brain over a 6-week duration at a flow rate of $0.15 \mu \mathrm{L} /$ hour. This dose of NLRP3 siRNA or caspase-1 siRNA infusion was well tolerated, and no signs of neurotoxicity including hind-limb paralysis, vocalization, food intake, or neuroanatomical damage were observed in preliminary study. 
Incidentally, the behavioral and biochemical data between control siRNA-treated and non-siRNA-treated rats do not differ. All of the operations were carried out with aseptic techniques.

\section{Electrode implantation and SE induction}

SE was triggered following the methods of our previous work [24]. Briefly, rats were fixed in a stereotactic apparatus (Stoelting, Wood Dale, IL, USA) under deep anesthesia (10\% chloral hydrate, $0.3 \mathrm{~mL} / 100 \mathrm{~g}$, intraperitoneal). The electrodes were permanently implanted into the right basolateral amygdala (AP: $-3.0 \mathrm{~mm}$; $:-4.8 \mathrm{~mm}$; V: $-8.8 \mathrm{~mm}$ ) and were connected to a miniature receptacle, embedding in the skull with screws and dental acrylic cement. After electrode implantation, the animals were allowed to recover from surgery for 2 weeks. SE was induced by continuous delivery of 100-ms trains, consisting of $60 \mathrm{~Hz} 400 \mu \mathrm{A}$ (peak-to-peak) bipolar 1-ms square-wave pulses, delivered at $60 \mathrm{~Hz}$ every 0.5 seconds using a ML1101 electronic stimulator (Nihon Kohden) via the implanted electrode for up to 20 minutes. Electroencephalograms (EEGs) of the right amygdala were recorded with a digital amplifier (AD Instrument, Bio Amp (Shanghai, China), USA). After 20 minutes of continuous stimulation the stimulation was interrupted and the behavioral and electrographic activity of the animals was observed for 60 seconds. If the behavior of the animals indicated the presence of epileptic activity (head nodding or limb clonus), observation was continued for another 5 minutes. If an animal did not meet the criteria of clonic SE (continuous EEG epileptiform spiking and recurrent clonic seizures), stimulation was resumed and the behavior of the animal was checked again after 5 minutes. Once the criteria of SE were achieved, no further stimulation was given. Stimulation period never exceeded 40 minutes. All of the operations were carried out with aseptic techniques. Sham rats were handled in the same manner but without receiving any electrical stimulation. Moreover, our preliminary experiments also show that this amygdala stimulation model is effective.

\section{Monitoring of SRS}

SRS occurrence was evaluated through a 4-week video monitoring started 2 weeks after SE. All recordings for SRS were done during the light period. Epileptic rats were video-recorded for at least 12 hours daily. The frequency and duration of stage 4/5 seizures were recorded, and the severity of SRS was scored according to Racine s scale [25]. The recordings were analyzed by observers who were blind to the results of group allocation.

\section{Brain tissue preparation}

Rats were sacrificed under deep anesthesia and were handled as follows: For Western blot analysis, quantitative real-time PCR, and ELISA, rats were perfused transcardially with $0.9 \%$ saline ( $\mathrm{pH} 7.4$ ) only. The brains were removed rapidly and stored in liquid nitrogen until use. For cresyl violet staining and terminal deoxynucleotidyl transferase-mediated dUTP end-labeling assay (TUNEL) analysis, rats were perfused transcardially with $0.9 \%$ saline ( $\mathrm{pH} 7.4$ ), followed by a fixative solution containing $4 \%$ paraformaldehyde in $0.9 \%$ saline ( $\mathrm{pH} 7.4$ ). The brains were removed and fixed in the same fixative at $4 \mathrm{C}$ until use. For double immunofluorescence staining, rat brain was removed without perfusion, embedded in tissue freezing medium, and immediately frozen at $-4 \mathrm{C}$. Frozen tissue was stored at $-80 \mathrm{C}$ until sectioning.

\section{Western blotting}

Tissues samples were digested with radio immunoprecipitation assay (RIPA) lysis buffer $(50 \mathrm{mmol} / \mathrm{L}$ Tris- $\mathrm{HCl}$, $150 \mathrm{mmol} / \mathrm{L} \mathrm{NaCl}, 1 \%$ Nonidet-40, 0.5\% sodium deoxycholate, $1 \mathrm{mmol} / \mathrm{L}$ EDTA, $1 \mathrm{mmol} / \mathrm{L}$ PMSF) with protease inhibitors (pepstatin $1 \mu \mathrm{g} / \mathrm{mL}$, aprotinin $1 \mu \mathrm{g} / \mathrm{mL}$, leupeptin $1 \mu \mathrm{g} / \mathrm{mL}$ ) for 30 minutes and centrifuged at 12,000 g for 15 minutes at $4 \mathrm{C}$. The protein concentration was determined using the Bradford assay kit (Bio-Rad Laboratories, Hercules, CA, USA). The equal amount of protein from different samples was separated using 8 to $12 \%$ sodium dodecyl sulfate (SDS) polyacrylamide gels and transferred to polyvinylidene fluoride (PVDF) membranes. The membranes were blocked with $10 \%$ non-fat milk in Tween-TBS (TBST) and incubated at $4 \mathrm{C}$ overnight, with the primary antibodies against NLRP3 (1:200; Santa Cruz Biotechnology, Santa Cruz, CA, USA), cleaved IL-1 $\beta$ (1:200; Santa Cruz Biotechnology, Santa Cruz, CA, USA), cleaved caspase-1 (1:200; Biorbyt, San Francisco, CA, USA), and $\beta$-actin (1:1,000; Santa Cruz Biotechnology, Santa Cruz, CA, USA). After rinsing, the membranes were appropriately incubated with horseradish peroxidase (HRP)-conjugated suitable secondary antibodies (1:5,000; Zhongshan Inc., Beijing, China) for 2 hours at room temperature. Cross-reactivity was visualized using electrochemiluminescence (ECL) Western blotting detection reagents and analyzed by scanning densitometry using a UVP BioDoc-It Imaging System (UVP, Upland, CA, USA).

\section{Quantitative Real-Time PCR}

Total RNA was extracted using TRIzol reagent (Invitrogen, Carlsbad, CA, USA), using the protocol supplied by the manufacturer. The cDNA was synthesized using Reverse Transcription System (Bio-Rad, Hercules, CA, USA). The reaction was performed at $42 \mathrm{C}$ for $50 \mathrm{mi}-$ nutes, $95 \mathrm{C}$ for 5 minutes, and $5 \mathrm{C}$ for 5 minutes, then the cDNA was stored at $-20 \mathrm{C}$. Amplification was carried out using the Stratagene Mx3000P real-time PCR system (Stratagene, La Jolla, CA, USA) and real-time SYBR Green PCR technology (Takara Bio, Inc., Shiga, 
Japan). Reverse transcription was performed in a final volume of $20 \mu \mathrm{L}$ containing $2 \mu \mathrm{L}$ cDNA, $10 \mu \mathrm{L}$ SYBR Green, $0.4 \mu \mathrm{L}$ ROX Reference Dye, $0.4 \mu \mathrm{L}$ forward and reverse primer $(1 \mathrm{~mol} / \mathrm{L})$, and $6.8 \mu \mathrm{L}$ nuclease-free water. The optimal conditions were 40 cycles of $95 \mathrm{C}$ for $30 \mathrm{sec}-$ onds, $60 \mathrm{C}$ for 32 seconds, and $72 \mathrm{C}$ for 30 seconds. All reactions were run in duplicates and the mean values are used. Total RNA concentrations from each sample were normalized by quantity of $\beta$-actin mRNA, and the target genes expression was evaluated by ratio of the number of target mRNA to $\beta$-actin mRNA. Relative expression of genes was obtained by the $2^{-\triangle \Delta C T}$ method. Primers were purchased from Invitrogen (Carlsbad, CA, USA) as follows (name: forward primer, reverse primer): nlrp3: 5 -cc agggctctgttcattg-3 , 5 -ccttggctttcacttcg-3; caspase-1: 5 -ag gagggaatatgtggg-3 , 5 -aaccttgggcttgtctt- $3 ; \beta$-actin: 5 -agg gaaatcgtgcgtgac 3,5 -cgctcattgccgatagtg-3 .

\section{Cytokine measurement}

The cytokine analysis was performed in duplicate using commercial ELISA assay kits according to the manufacturers instructions. IL- $1 \beta$ was measured in ELISAs from R\&D Systems (Minneapolis, MN, USA). IL-18 was measured in ELISAs from Invitrogen (Carlsbad, CA, USA). The results are expressed in $\mathrm{pg} / \mathrm{mL}$.

\section{Nissl staining (cresyl violet staining) and TUNEL staining}

The brains were embedded in paraffin and cut into $7-\mu \mathrm{m}$ sections. Nissl staining was employed to detect surviving neurons. Briefly, the paraffin-embedded sections were dewaxed and rehydrated according to the standard protocols, and immersed in $1 \%$ cresyl violet at $50 \mathrm{C}$ for 5 minutes. After being rinsed with water, the sections were dehydrated in increasing concentrations of ethanol, mounted on the slides, and examined with a light microscope. Only the neurons with a violet nucleus and intact morphology were counted as surviving neurons. The TUNEL staining, which detects DNA fragmentation resulting from apoptotic signaling cascades, was performed to label apoptotic neurons. It may also label cells that have suffered severe DNA damage. Therefore, the TUNEL assay is helpful in identifying seizure-induced neuronal loss in our experiment. We employed the TUNEL assay via a commercial kit according to the manufacturer's instructions (Roche Co., Mannheim, Germany). TUNEL-positive neurons with condensed nuclei were identified as dead neurons. Cell counting was performed on six randomly selected non-overlapping fields in the CA1 and CA3 regions of the hippocampus per slide. The densities of surviving neurons or TUNEL-positive neurons in the hippocampus of the scanned digital images were calculated using Image-Pro Express software (Media Cybernetics, Silver Spring, MD, USA). The total cell counts were averaged from six sections per animal. The survival index was defined as:

Surviving index \%

$$
\begin{gathered}
100 \times \text { Number of survivng neurons } \\
/ \text { Total numbers of neurons }
\end{gathered}
$$

Furthermore, the TUNEL-positive neuron index was defined as:

TUNEL-positive neurons index \%

$100 \times$ Count of TUNEL-positive neurons /Total count of neurons

\section{Double immunofluorescence staining}

In brief, frozen tissue sections of hippocampus area (20 $\mu \mathrm{m}$ thick) from epileptic rats at 12 hours following amygdala stimulation and time-matched shams were used for double staining of NLRP3/ionized $\mathrm{Ca}^{+}$binding adaptor molecule 1 (Iba1). The sections were obtained by cryosectioning at $-20 \mathrm{C}$, mounted on a glass slide, and incubated at room temperature for 1 hour. Afterward, the sections were fixed in ice-cold acetone for 10 minutes and then dried on a heater for 10 minutes at $40 \mathrm{C}$. The sections were then blocked with 5\% BSA and $0.1 \%$ Triton X-100 for 2 hours at room temperature. After a single wash with PBS, sections were incubated overnight at $4 \mathrm{C}$ with a goat polyclonal antibody against Iba1 (1:100, Abcam, Cambridge, UK) as well as a rabbit polyclonal antibody against NLRP3 (1:50; Santa Cruz Biotechnology, Santa Cruz, CA, USA)). Sections were rinsed in PBS and washed 3 times, and then incubated respectively with tetramethylrhodamine (TRITC)conjugated anti-goat IgG (1:200; Zhongshan Inc., Beijing, China) and fluorescein isothiocyanate (FITC)-conjugated anti-rabbit IgG (1:200; Santa Cruz Biotechnology) for 2 hours at room temperature in a dark and humidified container. After that, the sections were washed with PBS and sealed with a coverslip. The slides were analyzed with a fluorescence microscopy (Olympus, Tokyo, Japan). For nuclear staining we used 4',6-diamidino-2-phenylindole (DAPI). To ensure the specificity of the immunoblotting procedure, control experiments were performed in which the corresponding primary antibody was omitted. Under these conditions, no signal was observed.

\section{Statistical analysis}

Statistical analysis was carried out by SPSS software 17.0 (IBM Inc., Chicago, IL, USA). After confirming normal distribution with skewness and kurtosis statistic test, independent sample $t$-test or one-way analysis of variance (ANOVA) followed by the Bonferroni post hoc test used to analyze differences among groups. All data were presented as mean standard deviation (SD). $P<0.05$ was considered statistically significant. 


\section{Results}

Cleaved IL-1 $\beta$, NLRP3 and cleaved caspase-1 were up-regulated in SE rat

We first investigated whether the expression of IL-1 $\beta$ was altered in rat brain after SE which was induced by amygdala stimulation. Total proteins were extracted from the hippocampal regions of SE rats at different time points (including 3, 6, 12, 24 hours following SE) and of shams, and subjected to Western blot analysis. Because there was no significant difference in all detected variables among different sham groups according to our preliminary analysis, the samples of sham group were collected at 12 hours after the sham operation of amygdala stimulation (without any electrical stimulation). We found that the cleaved IL-1 $\beta$ levels of SE rats were significantly elevated compared to control samples during all time points studied (Figure 1A). In the SE group, cleaved IL-1 $\beta$ levels dramatically increased at 3 hours and reached a maximum at 12 hours (about 4 those of the sham group ) after $\mathrm{SE}(P<0.05 ; \mathrm{n}=6)$. Following this maximum, no statistically significant difference was observed in expression level of cleaved IL-1 $\beta$ among rat brains at 24 hours compared with that at 12 hours $(P=1.000 ; \mathrm{n}=6)$. Next, we analyzed the expression of hippocampal NLRP3

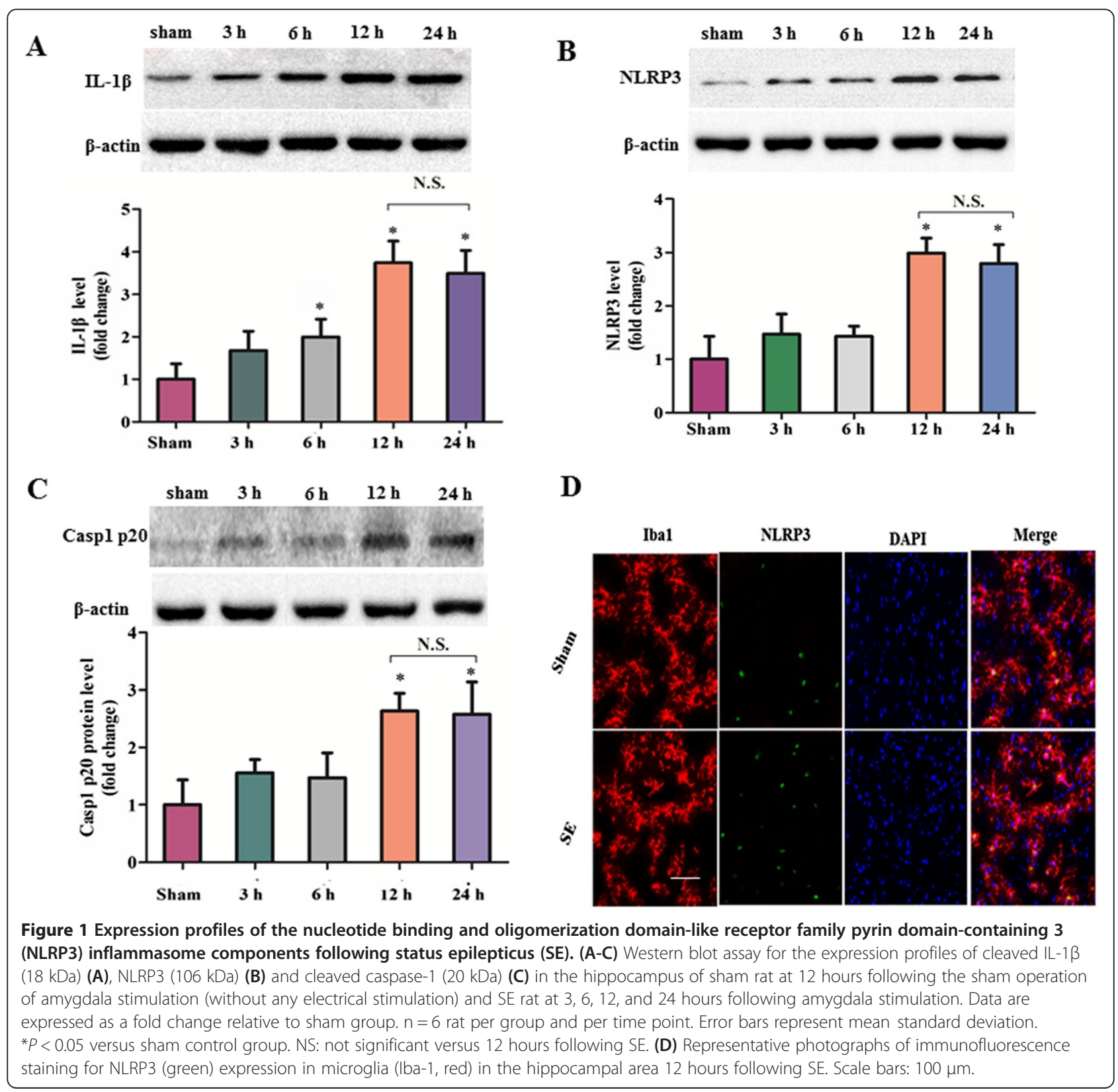


inflammasome components in SE rats. Importantly, the levels of hippocampal NLRP3 protein, and caspase-1 p20, the products of NLRP3 inflammasome activation, were significantly increased at 3 hours and reached a maximum at 12 hours after SE (both $P<0.05 ; \mathrm{n}=6$, Figure 1B, C). Accordingly, no significant differences were observed in NLRP3 levels $(P=1.000 ; \mathrm{n}=6)$, and cleaved caspase- 1 levels $(P=1.000 ; \mathrm{n}=6)$ among rat brains at 24 hours compared with that at 12 hours after SE. Using double immunofluorescence staining to colocalize NLRP3 with microglia marker Iba1, our result further demonstrated the increased expression of NLRP3 in the Iba1-positive microglia of hippocampus at 12 hours following SE (Figure 1D).
Downregulation of NLRP3 by siRNA led to significant reduction in proinflammatory cytokines and cleaved caspase-1 expression levels in SE rat

To directly study the potential impact of increased NLRP3 expression levels on the maturation and secretion of IL-1 $\beta$ in SE rats, we knocked down brain NLRP3 expression in animals before undergoing SE by using in-vivo nonviral RNA interference methodology [26]. To evaluate the silencing efficiency of siRNA infusion, the gene expression and protein level of NLRP3 protein were detected by quantitative real-time PCR and Western blotting, respectively. This approach of using NLRP3 siRNA infusion could produce a significant down-regulation of NLRP3 mRNA (by 60\%) and protein levels (by $51 \%$ ) in the

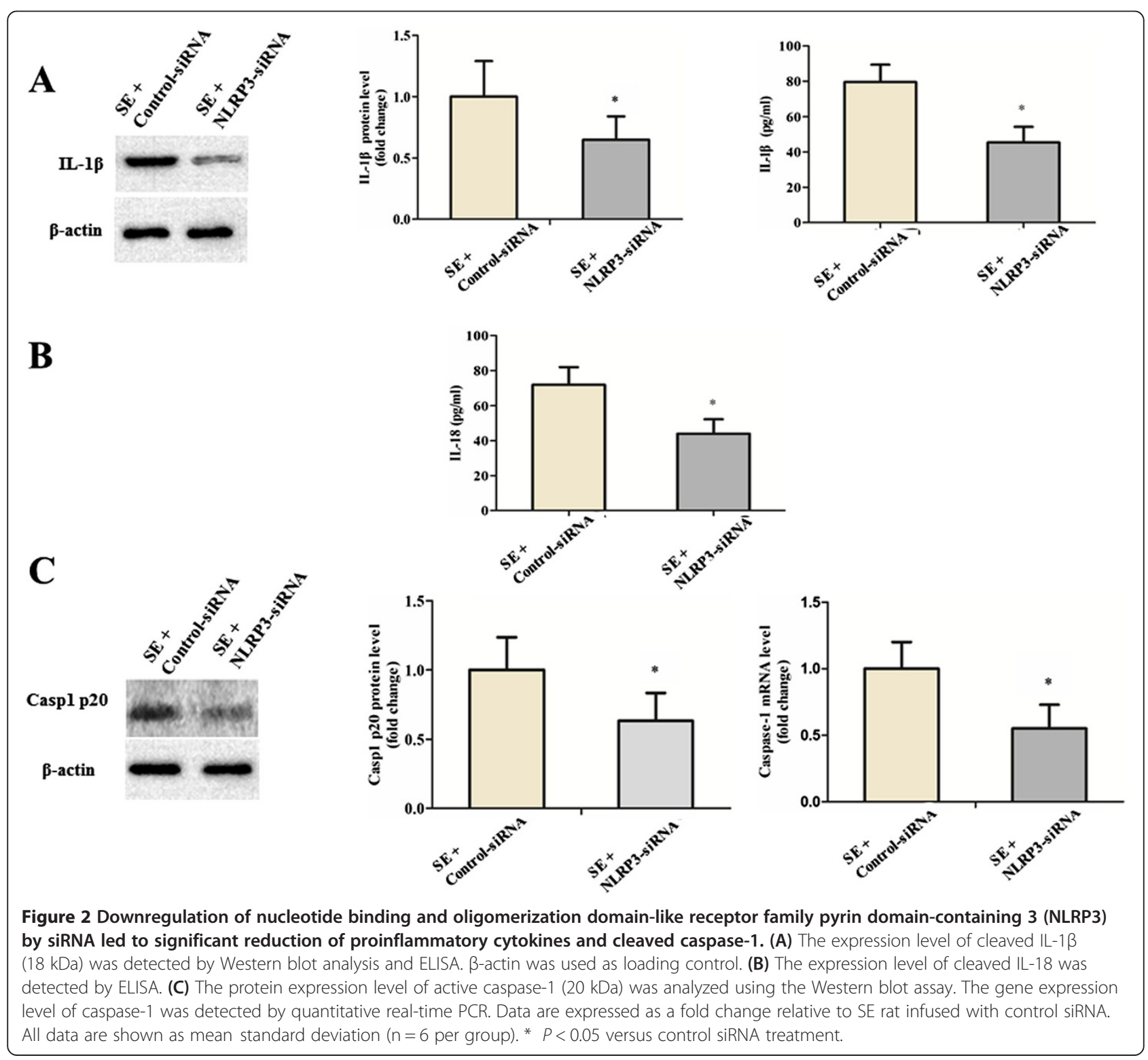


brain compared with control siRNA under epileptic conditions (all $P<0.05$; Additional file 2: Figure S2 A, B). Incidentally, the NLRP3 levels and gene expression between control-siRNA treated and non-siRNA-treated tissues under epileptic conditions or under control conditions do not differ (all $P>0.05 ; \mathrm{n}=6$, Additional file 2: Figure S2 A, $\mathrm{B}$, and $\mathrm{E})$, excluding potential indirect siRNA-effects on gene expression or the protein level of NLRP3.

Next, we determined the effects of NLRP3 knock down on SE-induced neuroinflammation. The proinflammatory cytokines expression levels were then assessed by Western blot analysis and ELISA assay. Compared to the controlsiRNA group, the elevated expression level of cleaved IL- $1 \beta$ in the brain tissues of SE rats could be inhibited by NLRP3 siRNA treatment $(P<0.05 ; \mathrm{n}=6$; Figure $2 \mathrm{~A})$. In addition, SE rats infused with NLRP3 siRNA showed a dramatic reduction in brain IL-18 protein levels $(P<0.05 ; \mathrm{n}=6$; Figure $2 \mathrm{~B})$. We further examined the changes in caspase-1, which has been known to play a central role in the cleavage of IL-1 $\beta$ and IL-18. The gene expression and protein level of caspase- 1 protein were detected by quantitative real-time PCR and Western blotting, respectively. As indicated in Figure $2 \mathrm{C}$, the NLRP3 siRNA treatment could reduce the active caspase1 protein levels and gene expression in SE rats (both $P<0.05 ; \mathrm{n}=6$ ).

\section{Downregulation of NLRP3 by siRNA attenuated the development and severity of spontaneous recurrent seizures following $\mathrm{SE}$}

In above work, we demonstrated that NLRP3 mediated IL-1 $\beta$ over-expression and inflammatory signal activation in the SE rat hippocampus. Accumulating data suggest that inflammation may contribute to epileptogenesis in experimental models as well as in humans. However, whether anti-inflammatory treatments can prevent epileptogenesis remains controversial. Here, we examined the anti-epileptogenic effect of NLRP3 inhibition.

To determine the effect of NLRP3 siRNA on the development and severity of SRS in the chronic phase, SRS were observed from a 4-week video recording, which started 2 weeks after SE. The severity of SRS between siRNA (non or control)-treated SE rats and SE rats do not differ (all $P>0.05 ; \mathrm{n}=18$, Additional file 3: Table S1), excluding an effect of siRNA transfection on status severity.

As revealed by Table 1(A), all rats (18/18) in the control siRNA-treated SE group had SRS during the monitoring period, whereas $66 \%(12 / 18)$ of the rats in the NLRP3 siRNA-treated SE group had SRS. The time to development of SRS was longer in the NLRP3 siRNA-treated SE group compared with the control siRNA-treated SE group (SE + control siRNA group: 14.85 .5 days; SE + NLRP3 siRNA group: 27.59 .3 days, Table 1A). The mean number of seizures (total number of seizures/number of recording days, calculated separately for each animal) in the control siRNA-treated SE group and NLRP3 siRNAtreated SE group was 7.581 .09 and 1.34 0.44 seizures/ day respectively. NLRP3 siRNA treatment also dramatically reduced the duration of observed seizures during the chronic epileptic phase (control siRNA, $26.626 .94 \mathrm{sec}-$ onds; NLRP3 siRNA, 10.482 .76 seconds, Table 1A). The differences between the two groups were significant $(P<0.05$, Table $1 \mathrm{~A})$.

NLRP3, nucleotide binding and oligomerization domainlike receptor family pyrin domain-containing 3; SE, status epilepticus; sec, seconds; SRS, spontaneous recurrent seizures.

Table 1 The development and severity of animals with spontaneous recurrent seizures (SRS) during the 4-week video monitoring in experimental groups

\begin{tabular}{|c|c|c|c|c|}
\hline \multicolumn{5}{|c|}{ (A) Results from rats after 6 weeks of treatment with NLRP3 siRNA or control siRNA } \\
\hline & $\begin{array}{l}\text { The number of rats } \\
\text { developing SRS }\end{array}$ & $\begin{array}{l}\text { The time to development } \\
\text { of SRS (days) }\end{array}$ & $\begin{array}{l}\text { The mean number of } \\
\text { seizures (seizures/day) }\end{array}$ & $\begin{array}{l}\text { The mean seizure } \\
\text { duration (sec/seizure) }\end{array}$ \\
\hline Control siRNA + sham & $0(18)$ & 0 & 0 & 0 \\
\hline NLRP3 siRNA+ sham & $0(18)$ & 0 & 0 & 0 \\
\hline Control siRNA + SE & $18(18)$ & 14.85 .5 & 7.581 .09 & 26.626 .94 \\
\hline NLRP3 SiRNA+ SE & $12(18)^{\mathrm{a}}$ & $27.59 .3^{a}$ & $1.340 .44^{a}$ & $10.482 .76^{\mathrm{a}}$ \\
\hline \multicolumn{5}{|c|}{ (B) Results from rats after 6 weeks of treatment with caspase-1 siRNA or control siRNA } \\
\hline & $\begin{array}{l}\text { The number of rats } \\
\text { developing SRS }\end{array}$ & $\begin{array}{l}\text { The time to development } \\
\text { of SRS (days) }\end{array}$ & $\begin{array}{l}\text { The mean number of } \\
\text { seizures (seizures/day) }\end{array}$ & $\begin{array}{l}\text { The mean seizure } \\
\text { duration (sec/seizure) }\end{array}$ \\
\hline Control siRNA + sham & $0(18)$ & 0 & 0 & 0 \\
\hline Caspase-1 siRNA + sham & $0(18)$ & 0 & 0 & 0 \\
\hline Control siRNA+ SE & $17(18)$ & 16.66 .1 & 9.021 .56 & 23.675 .90 \\
\hline Caspase-1 siRNA+ SE & $11(18)^{\mathrm{a}}$ & $26.89 .1^{a}$ & $2.400 .78^{a}$ & $8.212 .66^{a}$ \\
\hline
\end{tabular}

All data are shown as mean ? standard deviation ( $n=18$ per group). ${ }^{a} P<0.05$ versus control siRNA treatment. 


\section{Downregulation of NLRP3 by siRNA inhibited hippocampal neuronal loss in SE rat}

If left untreated, SE can cause irreversible brain damage. On this basis, we next investigated the effects of NLRP3 inhibition on neuronal loss in the CA1 and CA3 area of the hippocampus at 6 weeks after SE. Nissl staining was firstly used to detect surviving neurons in the hippocampus. As shown by Figure 3A, a significant increase in neuronal survival rate was noted in CA1 and CA3 regions in the hippocampus of SE rats treated with NLRP3 siRNA compared to the control-siRNA group (CA1: 77.34 5.7\% versus $59.575 .8 \%, \quad P<0.05$; CA3: $83.437 .3 \%$ versus $62.16 .5 \%, P<0.05)$. The TUNEL staining assay was then used. As indicated by Figure 3B, a dramatic reduction of the TUNEL-positive neurons index was observed in CA1 and CA3 regions of the hippocampus of SE rats treated with NLRP3 siRNA compared to the control-siRNA group (CA1: $11.412 .4 \%$ versus $15.953 .3 \%, \quad P<0.05$; CA3: 9.9 1.9\% versus $13.292 .8 \%, \quad P<0.05)$.

\section{Downregulation of caspase- 1 by siRNA alleviated} neuroinflammation, spontaneous recurrent seizures and hippocampal neuronal loss following SE

Considering the central role of caspase- 1 in the process of IL-1 $\beta$ maturation and secretion, we also successfully knocked down brain caspase-1 in SE rat by in-vivo nonviral RNA interference methodology for 6 weeks (Additional file 2: Figure S2 C, D, and F). We found a significant reduction of IL-1 $\beta$ levels after infused with caspase- 1 siRNA $(P<0.05 ; \mathrm{n}=6$, Figure $4 \mathrm{~A})$. Furthermore, caspase- 1 silencing attenuated the development and severity of SRS in the chronic phase $(P<0.05 ; \mathrm{n}=18$, Table $1 \mathrm{~B})$. A marked

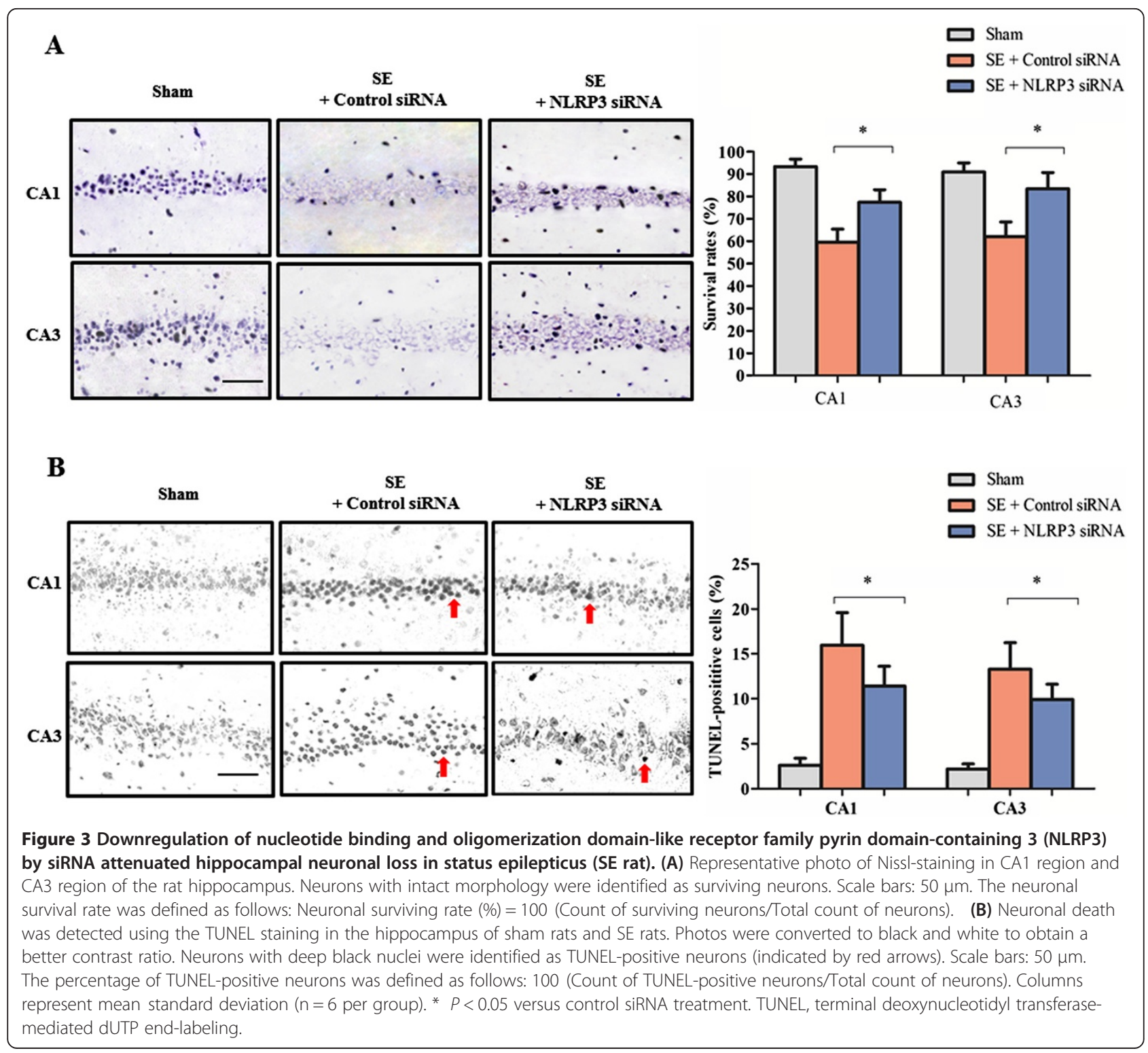




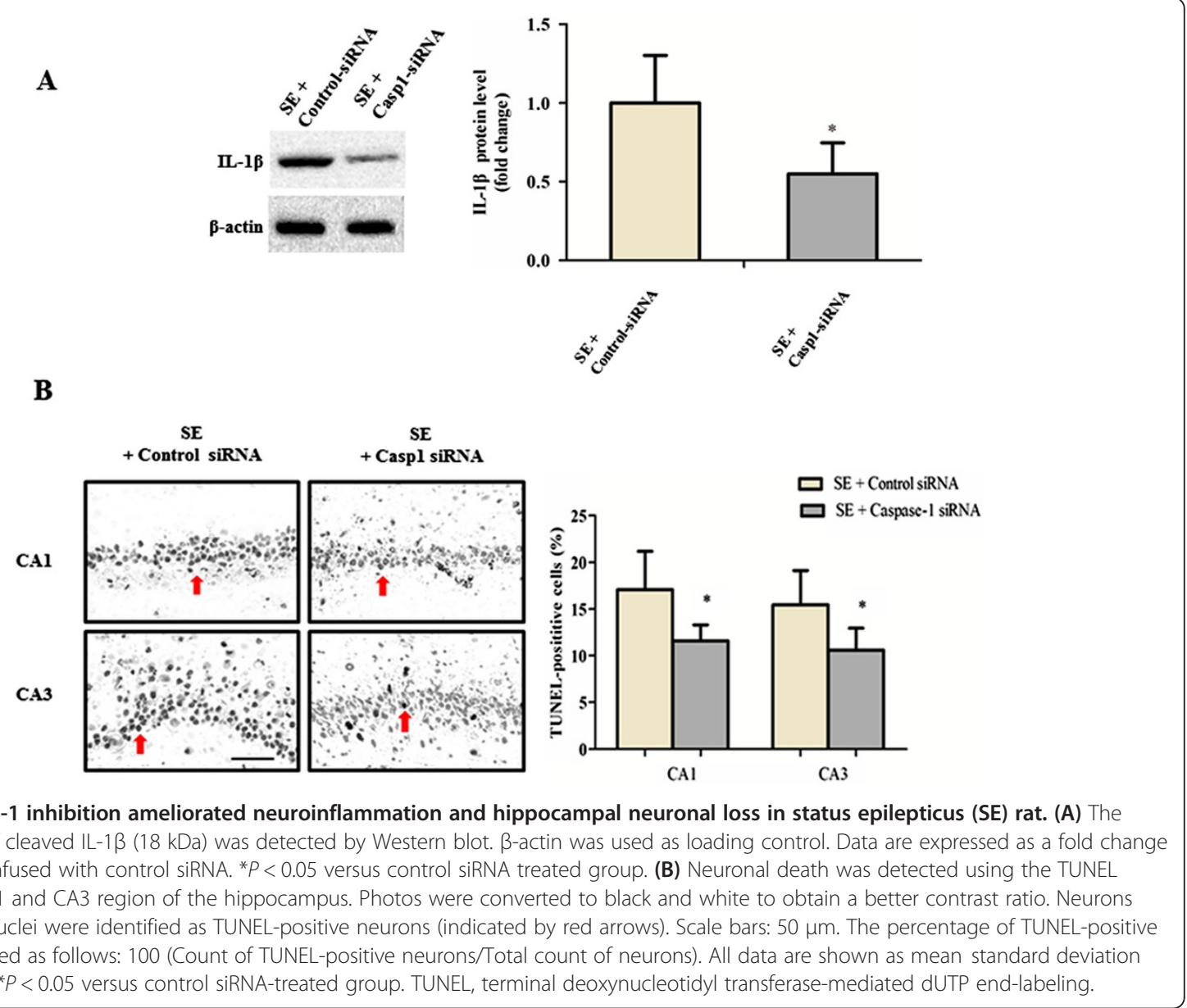

reduction of TUNEL-positive cell densities in the CA1 and $\mathrm{CA} 3$ region of the hippocampus in the SE rat treated with caspase- 1 siRNA was also observed $(P<0.05 ; \mathrm{n}=6$, Figure 4B).

\section{Discussion}

Experimental evidence supports a role for inflammatory processes in the precipitation and recurrence of seizures and neuronal damage. The involvement of proinflammatory cytokine IL-1 $\beta$ in the development of seizure is strongly supported by pharmacological and genetic studies in animal models, showing that interference with IL- $1 \beta$ reduces the incidence of SE or attenuates recurrent seizures $[8,27,28]$, whereas its amplification exacerbates seizures $[27,29,30]$ as well as lowers the seizure threshold $[15,31]$. Accordingly, selective blockade $[8,9]$, or gene deletion [9] of caspase-1, the enzyme which cleaves pro-IL-1 $\beta$ producing the mature and biologically active form of IL-1 $\beta$, reduces seizures significantly. IL- $1 \beta$ could also affect neuronal excitability at different levels [32 34]. Both hyperexcitability and excitotoxicity are required and need to be sufficient for the contributory effect of IL-1 $\beta$ to the generation of SRS. Moreover, specific cytokines, including
IL-1 $\beta$, have been shown to contribute to neuronal death [35], perhaps in part via enhanced excitability [5]. Conversely, SE, in the absence of pre-existing or concomitant systemic or CNS inflammation, induce proinflammatory reactions in the brain (that is sterile inflammation), which in turn contribute to seizure recurrence and severity [36].

Therefore, inflammation is both a cause and a consequence of SE. Experimental studies show that once SE develop, it can contribute to perpetuate inflammation in the brain, thus activating a vicious cycle that in turn fosters aberrant hyperexcitability (Figure 5). Thus, a prolonged condition of ongoing seizures with SE can be generated.

Our study was to examine for first time whether the NLRP3 inflammasome is a potential mechanism in neuroinflammation of SE rats. We compared expression of proinflammatory cytokine IL-1 $\beta$, NLRP3 and caspase-1 in hippocampus from SE rats model evoked by amygdala stimulation to matched sham samples. The present study detected up-regulated IL-1 $\beta$, NLRP3 and caspase-1 levels within SE samples than controls. All protein levels reach a maximum at 12 hours following SE. Meanwhile, the cellular localization of NLRP3 on microglia in SE rat brain was demonstrated by double immunofluorescence 


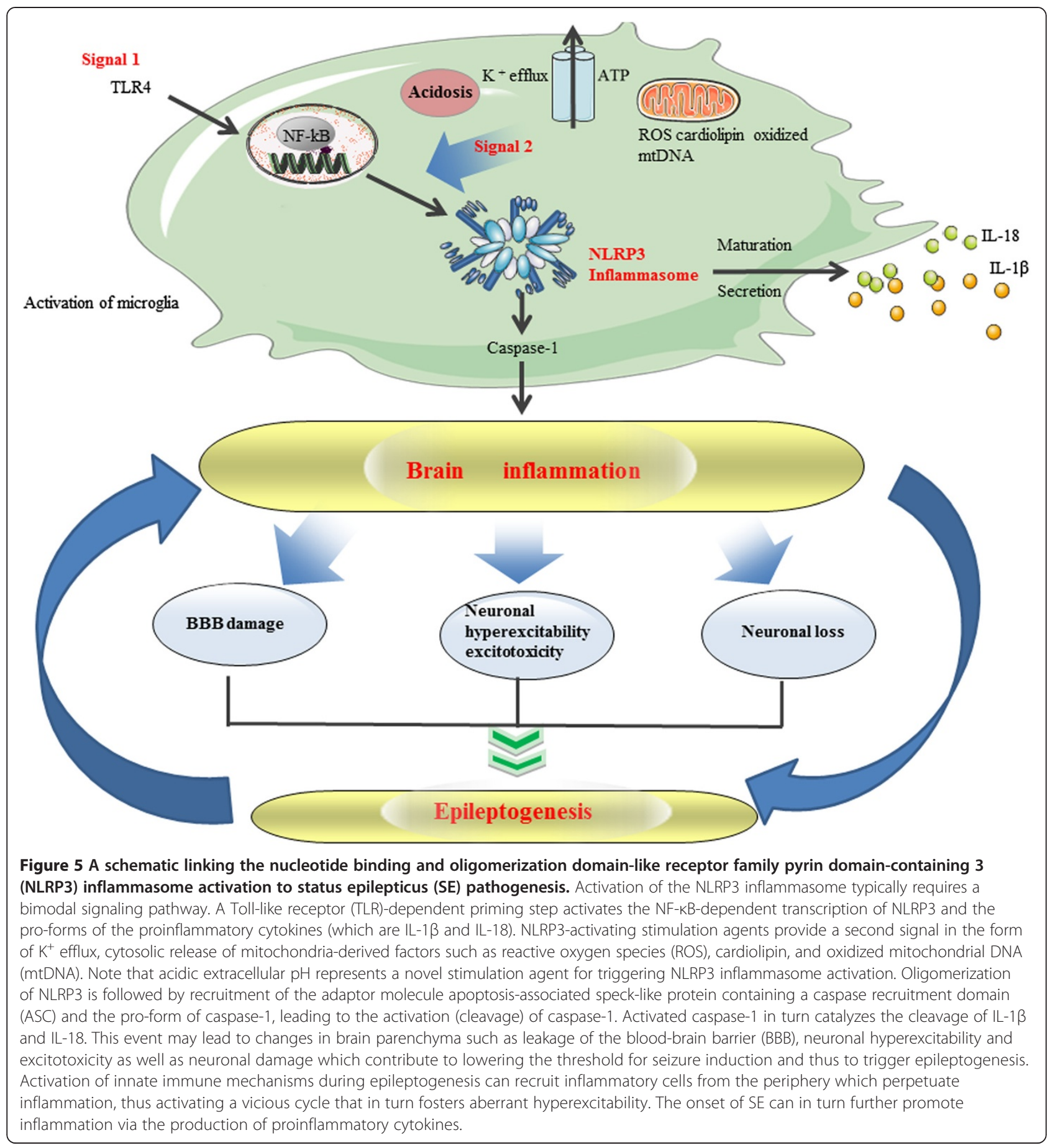

staining. Moreover, we knocked down brain NLRP3 expression by implanting mini-osmotic pumps for direct infusion of siRNA to investigate its role on neuroinflammation in the SE rat model. In our study, we found that this approach effectively down-regulated the levels of NLRP3 mRNA and protein in SE rat brain. Meanwhile, compared to non-siRNA-treated SE rat, the treatment with control siRNA did not alter NLRP3 mRNA and protein levels, thus excluding an effect of pump-mediated infusion on NLRP3 expression levels. For the first time, we revealed that NLRP3 siRNA treatment could significantly reduce proinflammatory cytokine levels and the active caspase-1 expression levels. Meanwhile, NLRP3 inhibition could also suppress SRS, and attenuate hippocampal neuronal loss.

As we know, caspase-1 is a critical pathway by which NLRP3 inflammasomes contribute to the downstream effects. Hence, we also knocked down brain caspase- 1 by this 
incpyvivo nonviral RNA interference methodology in SE rats. Consistent biochemical and behavioral results were found between caspase-1 siRNA- and NLRP3 siRNAtreated SE rats, further supporting that NLRP3 exerts the effects of neuroinflammation in rats following SE.

We have determined that the NLRP3 inflammasome contributes to the SE-induced inflammatory response; however, the molecular basis of NLRP3 inflammasome activation in SE-induced brain injury has not been established. Evidence shows that assembly of the NLRP3 inflammasome depends on the activation of NLRP3. This process relies on exposure to whole pathogens, as well as a number of structurally diverse pathogen or dangerassociated molecular patterns (PAMPs or DAMPs, respectively) and environmental irritants. It should be noted that, the high concentrations of extracellular ATP and $\mathrm{K}^{+}$ ions, and the generation of ROS are the most important factors for activating NLRP3 [37]. In fact, all of the factors above can be involved after SE. Additionally, it should be noted that IL-1 $\beta$ may trigger the classical cascade of events which includes the activation of the NF-kBdependent pathways, thus resulting in the transcription of genes that may contribute to the acquired molecular changes (for example, modifications in ion channels) associated with the epileptogenic process [38]. Interestingly, NLRP3, an important component of the NLRP3 inflammasome complex, is activated by IKK $\beta / N F-\kappa B$ [12]. The activation of the NLRP3 inflammasome, results in the secretion of bioactive IL- $1 \beta$, and subsequently triggers the activation of NF- $\mathrm{kB}$ which in turn promotes the secretion of bioactive IL-1 $\beta$ [39]. Local acidosis has been demonstrated at inflammatory sites. Recent data suggest that acidosis is a regulator of inflammatory pathways $[16,40]$. The study by Edye et al. [40] suggested that acidosis promotes alternative DAMP-induced processing of IL-1 $\beta$ independent of caspase- 1 , and this result seemed to be inconsistent with the findings by Rajamki and colleagues [16], as they found that acidic extracellular $\mathrm{pH}$ triggers NLRP3 inflammasome activation and IL-1 secretion in human macrophages (Figure 5).

\section{Conclusions}

Our study firstly demonstrates that the expression of the NLRP3 inflammasome was up-regulated in the SE rat. The increase in NLRP3 levels can activate caspase1 signaling that is responsible for neuroinflammation, neuronal loss and epileptogenesis (Figure 5). Using the pump-mediated in vivo infusion of nonviral siRNA to knock down NLRP3 and caspase-1 in the brain of SE rats, our study further indicated that inhibition of the NLRP3 inflammasome may play a neuroprotective role against SE-related neuroinflammation and neuronal damage.

\section{Additional files}

\begin{abstract}
Additional file 1: Figure S1. Scheme of the experimental design and the main experimental protocol. The rats were randomly divided into five groups: sham group, SE group, control siRNA + SE group, NLRP3 siRNA + SE group, and caspase-1 siRNA + SE group. siRNA, small interfering RNA; SE, status epilepticus; TUNEL assay, terminal deoxynucleotidyl transferasemediated dUTP end-labeling assay; Elisa, enzyme-linked immunosorbent assay; qRT-PCR, quantitative real-time PCR.
\end{abstract}

Additional file 2: Figure S2. Small interfering RNA (siRNA) targeting NLRP3 or caspase-1 effectively downregulated NLRP3 or caspase-1 in status epilepticus (SE) rat. (A) Messenger RNA levels of NLRP3 in brain of SE rats after 6-week infusion of artificial cerebrospinal fluid (aCSF), control SiRNA or NLRP3 siRNA. (B) Protein levels of NLRP3 in brain of SE rats after 6-week infusion of artificial cerebrospinal fluid (aCSF), control siRNA, or NLRP3 siRNA. (C) Messenger RNA levels of caspase-1 in brain of SE rats after 6-week infusion of aCSF, control siRNA, or caspase-1 siRNA. (D) Protein levels of caspase-1 in brain of SE rats after 6-week infusion of aCSF, control siRNA or caspase-1 siRNA. Data are expressed as a fold change relative to sham group. Columns represent mean standard deviation. $n=6$ rats per group. ${ }^{*} P<0.05$ versus control siRNA treatment. (E) Protein levels of NLRP3 in brain of sham rats after 6-week infusion of control siRNA, or aCSF. (F) Protein levels of caspase-1 in brain of sham rats after 6-week infusion of control siRNA oraCSF. Data are expressed as a fold change relative to sham group. Columns represent mean standard deviation. $n=6$ rats per group. NS: not significant versus sham rats.

Additional file 3: Table S1. Pump-mediated infusion of small interfering RNA (siRNA) prior to status epilepticus (SE) induction has no potential effects directly on the development and severity of spontaneous recurrent seizures (SRS).

\section{Abbreviations}

SE: status epilepticus; CNS: central nervous system; NLRP3: nucleotide binding and oligomerization domain-like receptor family pyrin domaincontaining 3; ASC: apoptosis-associated speck-like protein containing a caspase recruitment domain; siRNA: small interfering RNA; SDS: sodium dodecyl sulfate; PVDF: polyvinylidene fluoride; TRITC: tetramethylrhodamine; SRS: spontaneous recurrent seizures; PCR: polymerase chain reaction; ELISA: enzyme linked immunosorbent assay; TUNEL: terminal deoxynucleotidyl transferase-mediated dUTP end-labeling; RIPA: radio immunoprecipitation assay; EEG: Electroencephalogram; Iba1: ionized calcium binding adaptor molecule 1; BBB: blood brain barrier.

\section{Competing interests}

The authors declare that they have no competing interests.

\section{Authors contributions}

XFM, LT, and JTY were the main researchers in this study, and contributed to writing the manuscript. XFM, MML, MST, TJ, HFW and CCT planned the study, wrote the protocol, were involved in the genetic and clinical aspects of data analyses, and revised the manuscript. All authors read and approved the final manuscript for publication.

\section{Acknowledgments}

This work was supported by grants from the National Natural Science Foundation of China to LT $(81171209,81371406)$ and JTY (81000544), grants from the Shandong Provincial Natural Science Foundation to LT (ZR2011HZ001) and JTY (ZR2010HQ004), and the Medicine and Health Science Technology Development Project of Shandong Province to LT (2011WSA02018) and JTY (2011WSA02020).

\section{Author details}

'Department of Neurology, Qingdao Municipal Hospital, School of Medicine, Qingdao University, Qingdao, PR China. ${ }^{2}$ Department of Neurology, Qingdao Municipal Hospital, Nanjing Medical University, Nanjing, PR China.

${ }^{3}$ Department of Neurology, Qingdao Municipal Hospital, College of Medicine and Pharmaceutics, Ocean University of China, Qingdao, PR China.

${ }^{4}$ Department of Neurology, Nanjing First Hospital, Nanjing Medical University, 
Nanjing, PR China. ${ }^{5}$ Department of Neurology, Memory and Aging Center, University of California, San Francisco, CA, USA.

Received: 12 August 2014 Accepted: 2 December 2014

Published online: 17 December 2014

\section{References}

1. Gastaut H: Clinical and electroencephalographical classification of epileptic seizures. Epilepsia 1970, 11:102 113.

2. Vezzani A, French J, Bartfai T, Baram TZ: The role of inflammation in epilepsy. Nat Rev Neurol 2011, 7:31 40.

3. Dube C, Vezzani A, Behrens M, Bartfai T, Baram TZ: Interleukin-1 beta contributes to the generation of experimental febrile seizures. Ann Neurol 2005, 57:152 155.

4. Vezzani A, Friedman A, Dingledine RJ: The role of inflammation in epileptogenesis. Neuropharmacology 2013, 69:16 24.

5. Vezzani A, Baram TZ: New roles for interleukin-1 Beta in the mechanisms of epilepsy. Epilepsy Curr 2007, 7:45 50.

6. Gomez CD, Buijs RM, Sitges M: The anti-seizure drugs vinpocetine and carbamazepine, but not valproic acid, reduce inflammatory IL-1 beta and TNF-alpha expression in rat hippocampus. J Neurochem 2014, 130:770 779 .

7. Jeon KI, Xu X, Aizawa T, Lim JH, Jono H, Kwon DS, Abe J, Berk BC, Li JD, Yan C: Vinpocetine inhibits NF-kappaB-dependent inflammation via an IKK-dependent but PDE-independent mechanism. Proc Natl Acad Sci U S A 2010, 107:9795 9800.

8. Maroso M, Balosso S, Ravizza T, lori V, Wright Cl, French J, Vezzani A: Interleukin-1 beta biosynthesis inhibition reduces acute seizures and drug resistant chronic epileptic activity in mice. Neurotherapeutics 2011, 8:304 315 .

9. Ravizza T, Lucas SM, Balosso S, Bernardino L, Ku G, Noe F, Malva J, Randle JC, Allan S, Vezzani A: Inactivation of caspase-1 in rodent brain: a novel anticonvulsive strategy. Epilepsia 2006, 47:1160 1168

10. Haneklaus M, O'Neill LA, Coll RC: Modulatory mechanisms controlling the NLRP3 inflammasome in inflammation: recent developments. Curr Opin Immunol 2013, 25:40 45.

11. Cassel SL, Sutterwala FS: Sterile inflammatory responses mediated by the NLRP3 inflammasome. Eur J Immunol 2010, 40:607 611.

12. Bauernfeind FG, Horvath G, Stutz A, Alnemri ES, MacDonald K, Speert D, Fernandes-Alnemri T, Wu J, Monks BG, Fitzgerald KA, Hornung V, Latz E: Cutting edge: NF-kappaB activating pattern recognition and cytokine receptors license NLRP3 inflammasome activation by regulating NLRP3 expression. J Immunol 2009, 183:787 791

13. Liu HD, Li W, Chen ZR, Hu YC, Zhang DD, Shen W, Zhou ML, Zhu L, Hang $\mathrm{CH}$ : Expression of the NLRP3 inflammasome in cerebral cortex after traumatic brain injury in a rat model. Neurochem Res 2013, 38:2072 2083.

14. Ransohoff RM, Brown MA: Innate immunity in the central nervous system. J Clin Invest 2012, 122:1164 1171.

15. Jha S, Srivastava SY, Brickey WJ, locca H, Toews A, Morrison JP, Chen VS, Gris D, Matsushima GK, Ting JP: The inflammasome sensor, NLRP3, regulates CNS inflammation and demyelination via caspase- 1 and interleukin-18. J Neurosci 2010, 30:15811 15820.

16. Rajamaki K, Nordstrom T, Nurmi K, Akerman KE, Kovanen PT, Oorni K, Eklund KK: Extracellular acidosis is a novel danger signal alerting innate immunity via the NLRP3 inflammasome. J Biol Chem 2013, 288:13410 13419.

17. Fann DY, Lee SY, Manzanero S, Chunduri P, Sobey CG, Arumugam TV: Pathogenesis of acute stroke and the role of inflammasomes. Ageing Res Rev 2013, 12:941 966.

18. Schroder K, Tschopp J: The inflammasomes. Cell 2010, 140:821 832

19. Wasterlain CG, Fujikawa DG, Penix L, Sankar R: Pathophysiological mechanisms of brain damage from status epilepticus. Epilepsia 1993, 34 (Suppl 1):S37 S53.

20. Kim JE, Yeo SI, Ryu HJ, Kim MJ, Kim DS, Jo SM, Kang TC: Astroglial loss and edema formation in the rat piriform cortex and hippocampus following pilocarpine-induced status epilepticus. J Comp Neurol 2010, 518:4612 4628.

21. Sheen SH, Kim JE, Ryu HJ, Yang Y, Choi KC, Kang TC: Decrease in dystrophin expression prior to disruption of brain-blood barrier within the rat piriform cortex following status epilepticus. Brain Res 2011, 1369:173 183

22. Chen JW, Wasterlain CG: Status epilepticus: pathophysiology and management in adults. Lancet Neurol 2006, 5:246 256
23. Bjorling DE, Wang Z-Y: Estrogen and neuroinflammation. Urology 2001, 57:40 46

24. Sun Z, Yu JT, Jiang T, Li MM, Tan L, Zhang Q: Genome-wide microRNA profiling of rat hippocampus after status epilepticus induced by amygdala stimulation identifies modulators of neuronal apoptosis. PLoS One 2013, 8:e78375.

25. Racine RJ: Modification of seizure activity by electrical stimulation. Il Motor seizure Electroencephalogr Clin Neurophysiol 1972, 32:281 294.

26. Thakker DR, Hoyer D, Cryan JF: Interfering with the brain: use of RNA interference for understanding the pathophysiology of psychiatric and neurological disorders. Pharmacol Ther 2006, 109:413 438.

27. Vezzani A, Moneta D, Conti M, Richichi C, Ravizza T, De Luigi A, De Simoni MG, Sperk G, Andell-Jonsson S, Lundkvist J, Iverfeldt K, Bartfai T: Powerful anticonvulsant action of IL-1 receptor antagonist on intracerebral injection and astrocytic overexpression in mice. Proc Natl Acad Sci U S A 2000, 97:11534 11539.

28. Marchi N, Fan Q, Ghosh C, Fazio V, Bertolini F, Betto G, Batra A, Carlton E, Najm I, Granata T, Janigro D: Antagonism of peripheral inflammation reduces the severity of status epilepticus. Neurobiol Dis 2009, 33:171 181.

29. Balosso S, Maroso M, Sanchez-Alavez M, Ravizza T, Frasca A, Bartfai T, Vezzani A: A novel non-transcriptional pathway mediates the proconvulsive effects of interleukin-1beta. Brain 2008, 131:3256 3265.

30. Vezzani A, Conti M, De Luigi A, Ravizza T, Moneta D, Marchesi F, De Simoni MG: Interleukin-1beta immunoreactivity and microglia are enhanced in the rat hippocampus by focal kainate application: functional evidence for enhancement of electrographic seizures. J Neurosci 1999, 19:5054 5065.

31. Heida JG, Moshe SL, Pittman QJ: The role of interleukin-1 beta in febrile seizures. Brain Dev 2009, 31:388 393.

32. Hu S, Sheng WS, Ehrlich LC, Peterson PK, Chao CC: Cytokine effects on glutamate uptake by human astrocytes. Neuroimmunomodulation 2000, 7:153 159.

33. Bezzi P, Domerca M, Brambilla L, Galli R, Schols D, De Clerca E, Vescovi A, Bagetta G, Kollias G, Meldolesi J, Volterra A: CXCR4-activated astrocyte glutamate release via TNFalpha: amplification by microglia triggers neurotoxicity. Nat Neurosci 2001, 4:702 710

34. Wang S, Cheng Q, Malik S, Yang J: Interleukin-1 beta inhibits gammaaminobutyric acid type $A(G A B A(A))$ receptor current in cultured hippocampal neurons. J Pharmacol Exp Ther 2000, 292:497 504.

35. Loscher W: Animal models of epilepsy for the development of antiepileptogenic and disease-modifying drugs. A comparison of the pharmacology of kindling and post-status epilepticus models of temporal lobe epilepsy. Epilepsy Res 2002, 50:105 123.

36. Vezzani A, Balosso S, Ravizza T: The role of cytokines in the pathophysiology of epilepsy. Brain Behav Immun 2008, 22:797 803.

37. Walsh JG, Muruve DA, Power C: Inflammasomes in the CNS. Nat Rev Neurosci 2014, 15:84 97

38. Pitkanen A, Sutula TP: Is epilepsy a progressive disorder? Prospects for new therapeutic approaches in temporal-lobe epilepsy. Lancet Neurol 2002, 1:173 181

39. Grant RW, Dixit VD: Mechanisms of disease: inflammasome activation and the development of type 2 diabetes. Front Immunol 2013, 4:50

40. Edye ME, Lopez-Castejon G, Allan SM, Brough D: Acidosis drives damageassociated molecular pattern (DAMP)-induced interleukin-1 secretion via a caspase-1-independent pathway. J Biol Chem 2013, 288:30485 30494

doi:10.1186/s12974-014-0212-5

Cite this article as: Meng et al:: Inhibition of the NLRP3 inflammasome provides neuroprotection in rats following amygdala kindling-induced status epilepticus. Journal of Neuroinflammation 2014 11:212. 